\title{
Unfolded Protein Response
}

National Cancer Institute

\section{Source}

National Cancer Institute. Unfolded Protein Response. NCI Thesaurus. Code C118900.

A cellular stress response process that is activated by the accumulation of unfolded or misfolded proteins in the cell. 